\title{
Control of mRNA Translation in ALS Proteinopathy
}

\author{
Gianluca Cestra ${ }^{1,2}$, Simona Rossi ${ }^{3}$, Michela Di Salvio ${ }^{1,2}$ and Mauro Cozzolino ${ }^{3 *}$ \\ ${ }^{1}$ Institute of Biology and Molecular Pathology (IBPM), CNR, Rome, Italy, ${ }^{2}$ Department of Biology and Biotechnology Charles \\ Darwin, University of Rome "Sapienza", Rome, Italy, ${ }^{3}$ Institute of Translational Pharmacology (IFT), CNR, Rome, Italy
}

Cells robustly reprogram gene expression during stress generated by protein misfolding and aggregation. In this condition, cells assemble the bulk of mRNAs into translationally silent stress granules (SGs), while they sustain the translation of specific mRNAs coding for proteins that are needed to overcome cellular stress. Alterations of this process are deeply associated to neurodegeneration. This is the case of amyotrophic lateral sclerosis (ALS), a neurodegenerative disorder caused by a selective loss of motor neurons. Indeed, impairment of protein homeostasis as well as alterations of RNA metabolism are now recognized as major players in the pathogenesis of ALS. In particular, evidence shows that defective mRNA transport and translation are implicated. Here, we provide a review of what is currently known about altered mRNA translation in ALS and how this impacts on the ability of affected cells to cope with proteotoxic stress.

Keywords: RNA translation, stress granules, elF2 $\alpha$, amyotrophic lateral sclerosis (ALS), proteotoxic stress

\section{INTRODUCTION}

OPEN ACCESS

Edited by:

Angelo Poletti,

Università degli Studi di Milano, Italy

Reviewed by:

James C. Vickers,

University of Tasmania, Australia Alessandro Provenzani, University of Trento, Italy

*Correspondence:

Mauro Cozzolino mauro.cozzolino@ift.cnr.it

Received: 31 January 2017 Accepted: 10 March 2017 Published: 23 March 2017

Citation: Cestra G, Rossi S, Di Salvio M and Cozzolino M (2017) Control of mRNA

Translation in ALS Proteinopathy. Front. Mol. Neurosci. 10:85. doi: 10.3389/fnmol.2017.00085
Amyotrophic lateral sclerosis (ALS) is a progressive, adult-onset neurodegenerative disease. The most significant feature of ALS is the preferential loss of motor neurons from brain and spinal cord. Yet, a huge heterogeneity characterizes ALS manifestations, and it is now accepted that ALS is a complex multi-systemic syndrome with important aspects that overlap with other neurodegenerative disorders (Swinnen and Robberecht, 2014). A wealth of mechanisms has been suggested to cause ALS pathogenesis, such as protein aggregation, oxidative stress, mitochondrial damage, excitotoxicity and RNA dys-metabolism (Taylor et al., 2016). Although a big effort is still needed to clarify whether and to which extent each of these mechanisms play a role, key aspects of disease pathogenesis have clearly emerged.

The most relevant ALS genes give rise to proteins that accumulate as misfolded and/or aggregated species inside cells, both as wild-type or mutated forms. Indeed, protein aggregates are readily found in SOD1-, TDP-43-, FUS- and C9orf72-ALS, the most frequent genetic forms of the disease. Accordingly, impaired protein homeostasis, a condition that is almost invariably associated to misfolding and aggregation, has been frequently reported in ALS (Ruegsegger and Saxena, 2016). Moreover, an early and specific induction of endoplasmic reticulum (ER) stress has been proposed to explain the selective vulnerability of motor neurons (Ng et al., 2015; Sun et al., 2015), suggesting that these alterations might play a key role in disease pathogenesis. This conclusion is further supported by the evidence that a number of genes that participate to the autophagic and proteasomal control of protein degradation, such as ubiquilin-2 (Deng et al., 2011), sequestosome-1 (Fecto et al., 2011), optineurin (Maruyama et al., 2010), valosin-containing protein (VCP; Johnson et al., 2010), TBK1 (Freischmidt et al., 2015) and VAPB (Chen et al., 2010), are associated to ALS. 
FUS and TDP-43, as well as other ALS-related genes, have also a recognized role in the regulation of RNA metabolism, including RNA transcription and splicing, microRNA processing and mRNA stability, transport and translation (thoroughly reviewed in Ratti and Buratti, 2016). Additionally, the expanded G4C2 hexanucleotide repeat in the C9orf72 gene, the most frequent genetic mutation in ALS, might impact the proper regulation of RNA metabolism, as a result of both the accumulation of RNAs containing the expansion, that might disrupt the activity of a number of RNA binding proteins (RBPs), and the expansion-driven production of poly-dipeptides (dipeptide repeat proteins, DPRs), that accumulate in cells and affect the proper intracellular trafficking of RNAs (Wen et al., 2017). Although a specific step of RNA processing that is predominantly affected in motor neurons has not emerged so far, mRNA translation might represent a central process in ALS, as alterations in the control of translation might directly affect motor neuron functions and their ability to regulate translation in condition of proteotoxic stress.

\section{ALTERATIONS OF TRANSLATIONAL CONTROL IN ALS}

Protein synthesis is defined by the three classical phases that include initiation, elongation and termination (Aitken and Lorsch, 2012; Hinnebusch et al., 2016). Translation initiation is the rate-limiting step and the most tightly regulated stage, in which the two ribosomal subunits are assembled into $80 \mathrm{~S}$ ribosomes with methionyl-tRNA paired to the mRNA start codon. In the canonical, cap-dependent translation initiation, once the GTP-bound form of the eukaryotic initiation factor eIF2 is loaded with the methionyl-tRNA, it builds the ternary complex. This binds the small ribosome subunit and generates the $43 \mathrm{~S}$ preinitiation complex. Such $43 \mathrm{~S}$ complex is assembled on the $5^{\prime}$ mRNA methyl cap by the initiation factor eIF3, with the contribution of the polyA-binding protein and the eIF4F complex. This is a crucial step, as mechanisms that inhibit eIF4F assembly will in turn inhibit translation initiation. Once formed, the $43 \mathrm{~S}$ preinitiation complex walks along the mRNA searching for an initiation AUG codon positioned in the optimal sequence context that is necessary for the establishing of an effective $48 \mathrm{~S}$ preinitiation complex. Arrest of the scanning process is caused by the codon-anticodon recognition, which elicits eIF5B GAP activity and thereby promoting the hydrolysis of the GTP in the ternary complex. eIF2-GDP is detached from the ribosome and this eventually leads to the formation of the $80 \mathrm{~S}$ ribosomal complex. Subsequently, translation elongation factors $(\mathrm{eEF})$ are recruited to promote polypeptide chain extension until the cycle is ended by the action of stop codon-dependent termination factors. In this process, the alpha subunit of eIF2 has a critical role. Indeed, phosphorylation of eIF $2 \alpha$ by stressinduced kinases is the leading mechanism that inhibits the formation of functional translation initiation complexes and causes mRNA accumulation into stress granules (SGs; Spriggs et al., 2010), leading to translational repression of the bulk of mRNAs in favor of those mRNAs that are required to subside stress. Recent studies support an intimate relationship between
ALS pathogenesis and protein synthesis: several ALS causative mutations affect the process of mRNA translation; novel unconventional and still unveiled translational processes, such as repeat associated non-AUG (RAN) translation, contribute to the pathogenesis of ALS.

\section{Defects in mRNA Transport and Local Translation}

Modification of neuronal activity ultimately depends on the precise localization and turnover of synaptic proteins such as neurotransmitters, channels, adapters and signaling molecules. Synaptic accumulation of these proteins is also achieved by local translation of specific mRNAs peripherally transported by RNA granules (Krichevsky and Kosik, 2001): ribonucleoprotein complexes containing ribosomes and temporary translationally arrested mRNAs, which are locally released and translated in response to appropriate synaptic stimuli. Several RBPs play a pivotal role in the assembly and control of RNA transport granules, by assisting mRNAs in their way from the cell body to the periphery and modulating their stability and translation.

Evidence suggests a direct association of dysfunctional neuronal transport granules with ALS. Both TDP-43 and FUS are found in RNA transport granules (Kanai et al., 2004; Elvira et al., 2006), and ALS-linked mutant TDP-43 affect their trafficking (Alami et al., 2014). FMRP, a translational repressor causally linked to Fragile X Syndrome, also associates to RNA transport granules, and interacts with TDP-43. FMRP expression in Drosophila robustly modifies TDP-43-mediated neurodegeneration. Further, both FMRP and TDP-43 collaborate to repress the translation initiation of mRNAs encoding proteins that are essential for synapse formation and plasticity such as Rac1, GluR1 and Map1b (Majumder et al., 2012, 2016; Coyne et al., 2014, 2015; Romano et al., 2016). Although the localization of FUS in motor neuron synaptic terminals has not been unambiguously clarified, recent findings suggest an involvement of FUS in the regulation of local translation. In non-neuronal cells FUS binds to the tumor-suppressor protein adenomatous polyposis coli (APC), which forms ribonucleoprotein complexes for targeting mRNAs to cell protrusions, and is required for efficient local translation of transcripts associated to peripheral cellular processes (Yasuda et al., 2013). In our previous work, we demonstrated that FUS carrying ALS causative mutations physically and genetically interacts with Pur-alpha (Di Salvio et al., 2015), a DNA-RNA binding protein with several functions, including the targeting of mRNAs to neuronal dendrites, as part of RNA transport granules. In particular, Pur-alpha behaves as a regulator of SG assembly and, together with FUS, inhibits protein synthesis, participating to FUS toxicity (Di Salvio et al., 2015). Intriguingly, Pur-alpha also binds to G4C2 expanded repeats of C9orf72 gene and ameliorates G4C2-mediated neurodegeneration in Drosophila (Xu et al., 2013).

\section{RAN Dipeptides and mRNA Translation}

Secondary and tertiary structures in the $5^{\prime}$ UTR of mRNAs strongly affect translation initiation efficiency 
(Green et al., 2016). When these regions are placed downstream the start codon, they intensely increase translation initiation, either in the case of suboptimal AUG or even in the total absence of a start codon (Green et al., 2016). This is particularly relevant in RAN translation, where translation starts in the absence of start codon and proceeds at multiple reading frames generating repeat-containing proteins. Both strands of C9orf72 gene carrying expanded repeats are actively transcribed, and both corresponding RNAs are expected to form stable secondary structures. Accordingly, both transcripts generate different DPRs by RAN translation: GA, GR, PA, PR and GP. The molecular machinery that controls RAN translation still needs to be fully defined (Green et al., 2016). However, recent key findings indicate that it uses a conventional cap-dependent ribosomal scanning, but bypasses normal requirements for start codon selection (Kearse et al., 2016). The formation of DPRs is widely believed to play a relevant role in C9orf72 toxicity. In particular, DPRs have been associated with functional alterations of proteins belonging to RNA transport granules. By analyzing the DPR interactome, it has clearly emerged, in fact, that the more toxic dipeptides GR and PR specifically bind to RBPs of the axonal RNA transport granules and to proteins of the translational machinery (Tao et al., 2015; Kanekura et al., 2016; Lee et al., 2016). In particular, GR and PR affect two translation related pathways: they localize in the nucleolus, where they block ribosomal RNA synthesis, and they alter SG formation in the cytoplasm. Further, PR makes complexes with mRNAs and prevents the access of translation factors (Kanekura et al., 2016), evidence that underlines, once more, the strong association between alterations in protein synthesis and the pathogenesis of ALS.

\section{REGULATION OF TRANSLATION IN CONDITION OF PROTEOTOXIC STRESS}

\section{Stress Granules in ALS}

Several of the RBPs connected to ALS localize into SGs. These are membrane-less, hydrogel-like structures that store translationally arrested mRNAs that accumulate during cellular stress, and that rapidly disassemble when stress is removed, in a process generally referred as "SG dynamics". SGs are composed by polyadenylated mRNAs, translation initiation factors (eIFs), small ribosomal subunits and a great number of RBPs (Protter and Parker, 2016), including ALS-linked RBPs. Among the latter, FUS and TDP-43 represent the most notable example of proteins that shuttle from cell nuclei, where they predominantly reside, into cytosolic SGs in response to a vast array of stress stimuli. This ability is held by wild type forms of both TDP-43 and FUS, that readily localize into SGs in stress conditions, and by ALS-linked mutant forms, that show an enhanced propensity to coalesce into SGs (Bosco et al., 2010).

In addition to TDP-43 and FUS, also hnRNP A2/B1 and hnRNP A1 (heterogeneous nuclear ribonucleoprotein A2/B1 and A1), two RBPs whose mutations are associated to rare forms of familial ALS (Kim et al., 2013), are part of SGs under stress conditions. This suggests a role of these two hnRNPs in stress response that might be impaired, by still unknown mechanisms, by their mutations (Guil et al., 2006; Martinez et al., 2016). Further, Ataxin 2, an ALS-linked RBP that causes spinocerebellar ataxia type 2 (SCA2), is associated to SGs and might have a role in their assembly (Nonhoff et al., 2007; Kaehler et al., 2012). However, also non RBP-like ALS factors, that are not obviously expected to be part of SGs, are indeed found into these structures. Profilin1, an actin binding protein whose mutations are a rare cause of familial ALS, localizes into SGs upon induction (Figley et al., 2014). Furthermore, mutant forms of SOD1, which are associated to frequent forms of familial ALS, may relocalize to SGs. Mutant SOD1s bind G3BP1, an RBP that plays important roles in SGs dynamics, and affect its ability to bind RNAs, thus interfering with SG assembly. As a result, the formation of SGs in response to stress is delayed or impaired, with obvious consequences on cell viability (Gal et al., 2016). Notably, a similar scenario seems to operate in C9orf72-ALS, where GR and PR dipeptides promote the spontaneous assembly of SGs and a consequent inhibition of global protein translation (Wen et al., 2014; Kanekura et al., 2016; Lee et al., 2016). Both GR and PR interact with G3BP1, suggesting that the simple interaction with G3BP1 is sufficient to impair SG dynamics, as in the case of SOD1 mutations, and that the accumulation of DPRs into SGs could be not required to alter their function (Lee et al., 2016).

Therefore, SGs might strongly contribute to ALS pathogenesis. Yet, how this occurs is still unclear. The tendency of ALS-related proteins to accumulate into SGs might play a relevant role in the formation of cytosolic aggregates that mark the affected tissues in ALS patients. Indeed, the intrinsic propensity of many RBPs to reversibly associate to each other (King et al., 2012) might act as a seed for the nucleation of intracellular aggregates when prolonged stress and/or mutations occur (Li et al., 2013). Nevertheless, evidence also exists that aggregates might originate independently (Shelkovnikova et al., 2014).

However, the association of ALS proteins to SGs supports a model whereby the disease might be the consequence of a disturbed SG regulation in both unstressed conditions, when mutant ALS factors might induce an uncontrolled and sustained activation of SG-mediated translational repression, or under stress, when such a response, that is usually strictly and finely tuned, might escape this regulation. Three lines of evidence support this model. First, genes that are involved in SG regulation are potent modifiers of the toxicity of mutant ALS genes, including TDP-43, Profilin 1 and C9orf72, in yeast and Drosophila (Couthouis et al., 2011; Kim et al., 2014; Lee et al., 2016). Second, several ALS-linked genes have a physiological role in SG formation and dynamics, and the loss of these functions might be involved in ALS (Aulas et al., 2012, 2015). Last, mutant ALS proteins might interfere with chaperone-mediated clearance from SGs of DRiPs, Defective Ribosomal Products constituted by terminated polypeptides that are released by disassembling polysomes prior to SG formation. Altered clearance of these peptides might lead to a decreased rate of SG disassembly, which further indicates that ALS can be associated with a protracted state of stress and the consequences of such a state (Ganassi et al., 2016). 


\section{Role of elF2 $\alpha$ Phosphorylation in ALS Pathology}

Being the key factor in the regulation of SG formation upon stress conditions, eIF $2 \alpha$ has attracted a large interest as a potential target for interventions aimed at modulating the stress response that is induced in different neurodegenerative conditions where proteotoxic stress occurs. Following the activation of the Unfolded Protein Response (UPR) by high levels of unfolded or misfolded proteins, the PERK kinase phosphorylates eIF $2 \alpha$. This decreases the active pool of the ternary complex with the consequent inhibition of global translation. This pathway is indeed involved in ALS pathology, since ER stress markers, including phosphorylated eIF $2 \alpha$, have been detected in tissues from ALS patients and animal models (Matus et al., 2013). However, whether the sustained activation of this response represents a protective mechanism or has a pathological role is unclear. This issue is of crucial importance, as it might help in defining whether enhancing or suppressing eIF $2 \alpha$ phosphorylation might be effective in contrasting the disease. To clarify this issue, the effects of modulation of eIF $2 \alpha$ phosphorylation on neurodegeneration have been largely investigated in different disease models.

Genetic approaches have been used to modulate the eIF $2 \alpha-P$ levels in mice overexpressing mutated G85R-SOD1. In particular, PERK haploinsufficiency accelerates SOD1 misfolding, causes an earlier disease onset and shortens survival (Wang et al., 2011). Opposite effects with a marked amelioration of disease were obtained by expressing in G85R-SOD1 mice an inactive form of GADD34, the eIF2 $\alpha-\mathrm{P}$ specific phosphatase (Wang et al.,

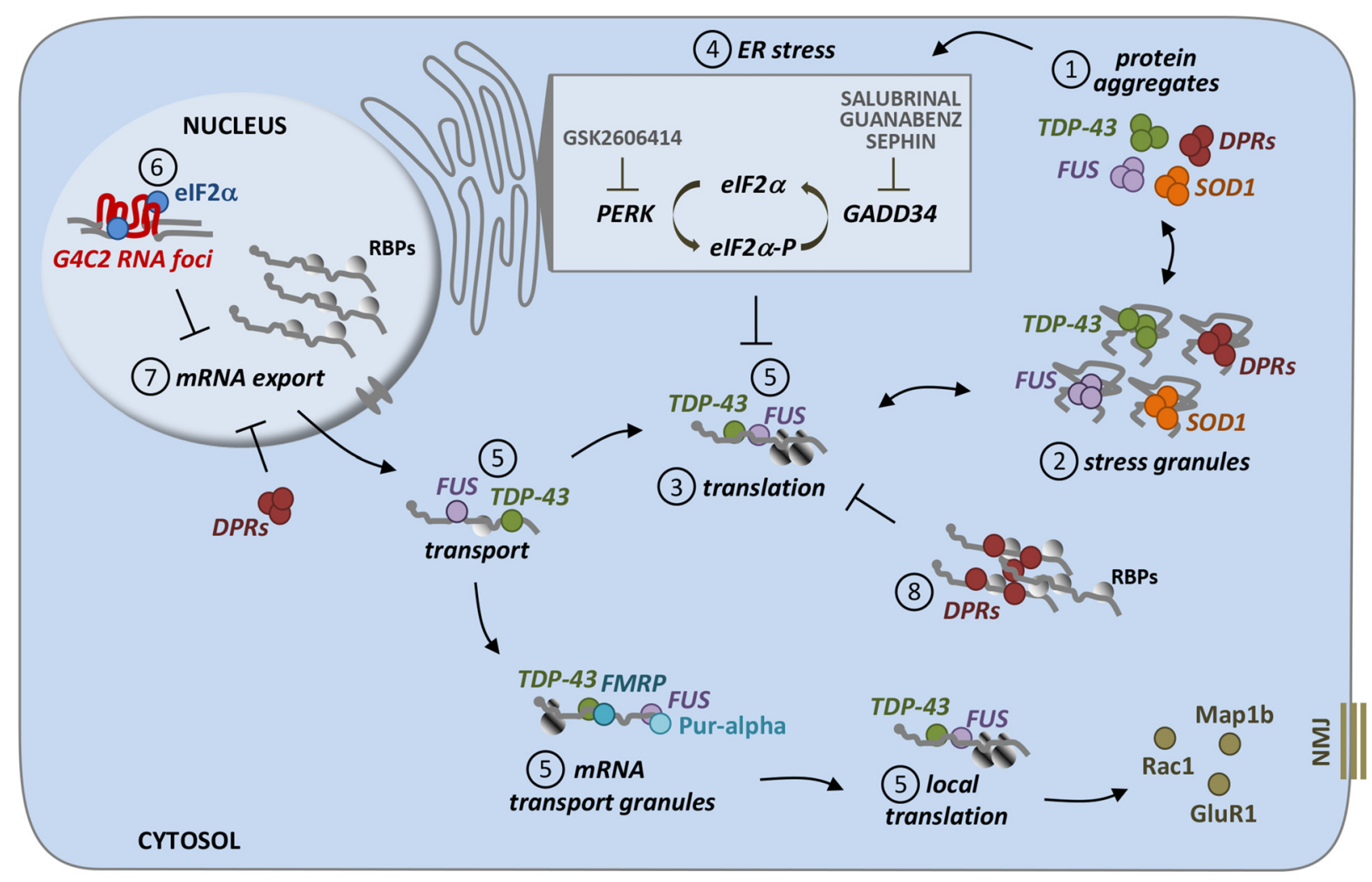

FIGURE 1 | Different pathways induced by ALS proteins converge on translational repression. Most of ALS proteins, including mutant SOD1, TDP-43, FUS, as well as DPRs (poly-dipeptide repeat proteins which are RAN-translated from G4C2 repeat expansion in C9orf72 gene), accumulate as misfolded and/or aggregated species inside cells (1). Interestingly, these protein aggregates are often found to be positive for stress granules markers suggesting a direct relationship between these two entities (2). SG formation is strongly associated to translational repression, which is indeed observed in ALS models linked to these genetic forms (3). Protein aggregates are able to induce ER stress, which is mediated by the activation of PERK kinase which in turn phosphorylates the translational initiation factor elF2a (elF2a-P; 4). elF2a-P has a central role in cell stress response, since it represses the translation of most cellular mRNA, while promoting the synthesis of proteins that help to overcome stress, including chaperones. Dephosphorylation of elF2a, mediated by the phosphatase GADD34, is then essential to turn off this pathway. Drugs such as GSK2606414, Salubrinal, Guanabenz and Sephin, that act on this mechanism have been tested in models for their therapeutic efficacy, giving conflicting results. Other ways, which are independent from elF2a phosphorylation, might be responsible of translational repression induced by ALS proteins. Indeed, FUS and TDP-43 are both RNA-binding proteins involved in RNA transport as part of RNA transport granules, and ALS-associated mutations might affect the proper localization and translation of specific mRNAs (such as Rac1, GluR1 and Map1b) that are important for neuromuscular junction (NMJ) structure and function (5). Moreover, C9orf72 RNA foci sequester elF2a, which might be less available for mRNA translation, thus resulting in translational inhibition and stress response (6). Finally, both repeat RNA foci and DPRs cause mRNA export defects, thus leading to the retention of mRNAs in cell nuclei and their reduced cytoplasmic availability (7). DPRs have been also found to directly bind mRNAs, making them less accessible to translation initiation factors, thus inhibiting protein translation (8). 
2014a). In line with these results, pharmacological treatment of SOD1-G93A mice with Salubrinal, Guanabenz, or its derivative Sephin, all inhibitors of eIF $2 \alpha$ de-phosphorylation, delays disease progression and prolongs survival (Saxena et al., 2009; Wang et al., 2014b; Das et al., 2015). Altogether, these data indicate that UPR has an important role in SOD1-mediated ALS, and support the idea that the induction of UPR and the prolonged activation of eIF $2 \alpha$ phosphorylation are beneficial to motor neuron survival, which is in line with the notion that stress response is essential for cell survival. However, in Drosophila the knockdown of PERK or GADD34 fly homologs respectively suppresses or enhances motility defects caused by TDP-43 toxicity (Kim et al., 2014). Moreover, a significant rescue of TDP-43 toxicity is observed in Drosophila and in primary rat cortical neurons followed by treatment with a specific PERK inhibitor (GSK2606414; Kim et al., 2014). The inhibition of the UPR pathway is also beneficial in other misfolding-related diseases, such as Prion Disease, Frontotemporal Dementia and Alzheimer (Moreno et al., 2012, 2013; Ma et al., 2013; Devi and Ohno, 2014; Radford et al., 2015). Taken together, these observations converge on the idea that while a transient eIF2 $\alpha$ phosphorylation is essential to overcome stress conditions, the persistent expression of eIF $2 \alpha-\mathrm{P}$ and the associated translational repression could be detrimental for neuronal viability and contribute to the pathogenic mechanisms. Moreover, they indicate that in ALS other pathways that converge on the regulation of protein translation might be activated together with, or even independently from misfolding-induced stress and might explain the different response to eIF $2 \alpha-\mathrm{P}$ targeting. In particular, this is suggested by the actual role that ALS factors such as TDP-43 and FUS might have in the control of protein translation, as previously mentioned, but also by the observation that stress response seems to be readily activated in C9orf72 ALS even in the absence of a clear phosphorylation of eIF $2 \alpha$. Indeed, accumulation of C9orf72 repeats causes a general

\section{REFERENCES}

Aitken, C. E., and Lorsch, J. R. (2012). A mechanistic overview of translation initiation in eukaryotes. Nat. Struct. Mol. Biol. 19, 568-576. doi: 10.1038/ns mb. 2303

Alami, N. H., Smith, R. B., Carrasco, M. A., Williams, L. A., Winborn, C. S., Han, S. S., et al. (2014). Axonal transport of TDP-43 mRNA granules is impaired by ALS-causing mutations. Neuron 81, 536-543. doi: 10.1016/j. neuron.2013.12.018

Aulas, A., Caron, G., Gkogkas, C. G., Mohamed, N. V., Destroismaisons, L., Sonenberg, N., et al. (2015). G3BP1 promotes stress-induced RNA granule interactions to preserve polyadenylated mRNA. J. Cell Biol. 209, 73-84. doi: $10.1083 /$ jcb.201408092

Aulas, A., Stabile, S., and Vande Velde, C. (2012). Endogenous TDP-43, but not FUS, contributes to stress granule assembly via G3BP. Mol. Neurodegener. 7:54. doi: 10.1186/1750-1326-7-54

Bosco, D. A., Lemay, N., Ko, H. K., Zhou, H., Burke, C., Kwiatkowski, T. J., et al. (2010). Mutant FUS proteins that cause amyotrophic lateral sclerosis incorporate into stress granules. Hum. Mol. Genet. 19, 4160-4175. doi: $10.1093 / \mathrm{hmg} / \mathrm{ddq} 335$

Chen, H. J., Anagnostou, G., Chai, A., Withers, J., Morris, A., Adhikaree, J., et al. (2010). Characterization of the properties of a novel mutation in VAPB impairment in nucleo-cytosol shuttling of RNAs (Freibaum et al., 2015; Rossi et al., 2015), leading to an entrapment in cell nuclei of polyadenylated mRNAs, which would be therefore less available for cytosolic translation. Interestingly, similar conclusions were drawn in cultured cells expressing argininecontaining polypeptides (PR and GR), that bind mRNAs and interfere with eIF4F assembly, thus inhibiting protein translation in the absence of enhanced eIF $2 \alpha$ phosphorylation (Kanekura et al., 2016).

In conclusion, an increasing amount of genetic and mechanistic indications point to alterations in the proper control of mRNA translation as critical players in ALS pathogenesis (Figure 1). Clearly, further investigations are needed to better define whether and to which extent protein misfolding and aggregation are the actual trigger of these events, how mutations affect the ability of ALS-linked genes to regulate mRNA transport and local protein synthesis, and how the therapeutic potential of targeting mRNA translation regulation can be converted into an effective treatment in different ALS conditions.

\section{AUTHOR CONTRIBUTIONS}

GC and MC formulated the concept of the manuscript. GC, SR and MC wrote the manuscript. SR and MDS executed complete drawing of the figure. All authors reviewed the manuscript.

\section{ACKNOWLEDGMENTS}

SR is recipient of a fellowship from Fondazione Umberto Veronesi. The following grants are gratefully acknowledged: Ministero dell'Economia, FaReBio di Qualità (GC); Ministero degli Affari Esteri e della Cooperazione, CHEMCHAP (GC); Ministero dell'Istruzione, Università e Ricerca, PRIN 2015-LFPNMN (MC); ARiSLA Foundation, project "FUSMALS" (MC). We apologize to our colleagues whose work was not included due to space restriction.

in familial amyotrophic lateral sclerosis. J. Biol. Chem. 285, 40266-40281. doi: 10.1074/jbc.M110.161398

Couthouis, J., Hart, M. P., Shorter, J., DeJesus-Hernandez, M., Erion, R., Oristano, R., et al. (2011). A yeast functional screen predicts new candidate ALS disease genes. Proc. Natl. Acad. Sci. US A 108, 20881-20890. doi: 10.1073/pnas. 1109434108

Coyne, A. N., Siddegowda, B. B., Estes, P. S., Johannesmeyer, J., Kovalik, T., Daniel, S. G., et al. (2014). Futsch/MAP1B mRNA is a translational target of TDP-43 and is neuroprotective in a Drosophila model of amyotrophic lateral sclerosis. J. Neurosci. 34, 15962-15974. doi: 10.1523/JNEUROSCI.252614.2014

Coyne, A. N., Yamada, S. B., Siddegowda, B. B., Estes, P. S., Zaepfel, B. L., Johannesmeyer, J. S., et al. (2015). Fragile X protein mitigates TDP-43 toxicity by remodeling RNA granules and restoring translation. Hum. Mol. Genet. 24 , 6886-6898. doi: 10.1093/hmg/ddv389

Das, I., Krzyzosiak, A., Schneider, K., Wrabetz, L., D’Antonio, M., Barry, N., et al (2015). Preventing proteostasis diseases by selective inhibition of a phosphatase regulatory subunit. Science 348, 239-242. doi: 10.1126/science.aaa4484

Deng, H.-X., Chen, W., Hong, S.-T., Boycott, K. M., Gorrie, G. H., Siddique, N., et al. (2011). Mutations in UBQLN2 cause dominant X-linked juvenile and adult-onset alS and ALS/dementia. Nature 477, 211-215. doi: 10.1038/nature10353 
Devi, L., and Ohno, M. (2014). PERK mediates eIF2 $\alpha$ phosphorylation responsible for BACE1 elevation, CREB dysfunction and neurodegeneration in a mouse model of Alzheimer's disease. Neurobiol. Aging 35, 2272-2281. doi: 10.1016/j. neurobiolaging.2014.04.031

Di Salvio, M., Piccinni, V., Gerbino, V., Mantoni, F., Camerini, S., Lenzi, J., et al. (2015). Pur-alpha functionally interacts with FUS carrying ALS-associated mutations. Cell Death Dis. 6:e1943. doi: 10.1038/cddis.2015.295

Elvira, G., Wasiak, S., Blandford, V., Tong, X. K., Serrano, A., Fan, X., et al. (2006). Characterization of an RNA granule from developing brain. Mol. Cell. Proteomics 5, 635-651. doi: 10.1074/mcp.M500255-MCP200

Fecto, F., Yan, J., Vemula, S. P., Liu, E., Yang, Y., Chen, W., et al. (2011). SQSTM1 mutations in familial and sporadic amyotrophic lateral sclerosis. Arch. Neurol. 68, 1440-1446. doi: 10.1001/archneurol.2011.250

Figley, M. D., Bieri, G., Kolaitis, R. M., Taylor, J. P., and Gitler, A. D. (2014). Profilin 1 associates with stress granules and ALS-linked mutations alter stress granule dynamics. J. Neurosci. 34, 8083-8097. doi: 10.1523/JNEUROSCI.054314.2014

Freibaum, B. D., Lu, Y., Lopez-Gonzalez, R., Kim, N. C., Almeida, S., Lee, K. H., et al. (2015). GGGGCC repeat expansion in C9ORF72 compromises nucleocytoplasmic transport. Nature 525, 129-133. doi: 10.1038/nature 14974

Freischmidt, A., Wieland, T., Richter, B., Ruf, W., Schaeffer, V., Müller, K., et al. (2015). Haploinsufficiency of TBK1 causes familial ALS and fronto-temporal dementia. Nat. Neurosci. 18, 631-636. doi: 10.1038/nn.4000

Gal, J., Kuang, L., Barnett, K. R., Zhu, B. Z., Shissler, S. C., Korotkov, K. V., et al. (2016). ALS mutant SOD1 interacts with G3BP1 and affects stress granule dynamics. Acta Neuropathol. 132, 563-576. doi: 10.1007/s00401-016-1601-x

Ganassi, M., Mateju, D., Bigi, I., Mediani, L., Poser, I., Lee, H. O., et al. (2016). A surveillance function of the HSPB8-BAG3-HSP70 chaperone complex ensures stress granule integrity and dynamism. Mol. Cell 63, 796-810. doi: 10.1016/j. molcel.2016.07.021

Green, K. M., Linsalata, A. E., and Todd, P. K. (2016). RAN translation-What makes it run? Brain Res. 1647, 30-42. doi: 10.1016/j.brainres.2016.04.003

Guil, S., Long, J. C., and Cáceres, J. F. (2006). hnRNP A1 relocalization to the stress granules reflects a role in the stress response. Mol. Cell. Biol. 26, 5744-5758. doi: 10.1128/mcb.00224-06

Hinnebusch, A. G., Ivanov, I. P., and Sonenberg, N. (2016). Translational control by $5^{\prime}$-untranslated regions of eukaryotic mRNAs. Science 352, 1413-1416. doi: $10.1126 /$ science.aad 9868

Johnson, J. O., Mandrioli, J., Benatar, M., Abramzon, Y., Van Deerlin, V. M., Trojanowski, J. Q., et al. (2010). Exome sequencing reveals VCP mutations as a cause of familial ALS. Neuron 68, 857-864. doi: 10.1016/j.neuron.2010. 11.036

Kaehler, C., Isensee, J., Nonhoff, U., Terrey, M., Hucho, T., Lehrach, H., et al. (2012). Ataxin-2-like is a regulator of stress granules and processing bodies. PLoS One 7:e50134. doi: 10.1371/journal.pone.0050134

Kanai, Y., Dohmae, N., and Hirokawa, N. (2004). Kinesin transports RNA: isolation and characterization of an RNA-transporting granule. Neuron 43, 513-525. doi: 10.1016/j.neuron.2004.07.022

Kanekura, K., Yagi, T., Cammack, A. J., Mahadevan, J., Kuroda, M., Harms, M. B., et al. (2016). Poly-dipeptides encoded by the C9ORF72 repeats block global protein translation. Hum. Mol. Genet. 25, 1803-1813. doi: $10.1093 / \mathrm{hmg} / \mathrm{ddw} 052$

Kearse, M. G., Green, K. M., Krans, A., Rodriguez, C. M., Linsalata, A. E., Goldstrohm, A. C., et al. (2016). CGG repeat-associated non-AUG translation utilizes a cap-dependent scanning mechanism of initiation to produce toxic proteins. Mol. Cell 62, 314-322. doi: 10.1016/j.molcel.2016.02.034

Kim, H. J., Kim, N. C., Wang, Y. D., Scarborough, E. A., Moore, J., Diaz, Z., et al. (2013). Mutations in prion-like domains in hnRNPA2B1 and hnRNPA1 cause multisystem proteinopathy and ALS. Nature 495, 467-473. doi: 10.1038 /nature 11922

Kim, H.-J., Raphael, A. R., LaDow, E. S., McGurk, L., Weber, R. A., Trojanowski, J. Q., et al. (2014). Therapeutic modulation of eIF2 $\alpha$ phosphorylation rescues TDP-43 toxicity in amyotrophic lateral sclerosis disease models. Nat. Genet. 46, 152-160. doi: 10.1038/ng.2853

King, O. D., Gitler, A. D., and Shorter, J. (2012). The tip of the iceberg: RNA-binding proteins with prion-like domains in neurodegenerative disease. Brain Res. 1462, 61-80. doi: 10.1016/j.brainres.2012.01.016
Krichevsky, A. M., and Kosik, K. S. (2001). Neuronal RNA granules: a link between RNA localization and stimulation-dependent translation. Neuron 32, 683-696. doi: 10.1016/S0896-6273(01)00508-6

Lee, K.-H., Zhang, P., Kim, H. J., Mitrea, D. M., Sarkar, M., Freibaum, B. D., et al. (2016). C9orf72 dipeptide repeats impair the assembly, dynamics, and function of membrane-less organelles. Cell 167, 774-788.e17. doi: 10.1016/j.cell.2016. 10.002

Li, Y. R., King, O. D., Shorter, J., and Gitler, A. D. (2013). Stress granules as crucibles of ALS pathogenesis. J. Cell Biol. 201, 361-372. doi: 10.1083/jcb. 201302044

Ma, T., Trinh, M. A., Wexler, A. J., Bourbon, C., Gatti, E., Pierre, P., et al. (2013). Suppression of eIF2 $\alpha$ kinases alleviates Alzheimer's disease-related plasticity and memory deficits. Nat. Neurosci. 16, 1299-1305. doi: 10.1038/nn.3486

Majumder, P., Chen, Y.-T., Bose, J. K., Wu, C.-C., Cheng, W.-C., Cheng, S.-J., et al. (2012). TDP-43 regulates the mammalian spinogenesis through translational repression of Rac1. Acta Neuropathol. 124, 231-245. doi: 10.1007/s00401-0121006-4

Majumder, P., Chu, J. F., Chatterjee, B., Swamy, K. B., and Shen, C. J. (2016). Co-regulation of mRNA translation by TDP-43 and Fragile X syndrome protein FMRP. Acta Neuropathol. 132, 721-738. doi: 10.1007/s00401-0161603-8

Martinez, F. J., Pratt, G. A., Van Nostrand, E. L., Batra, R., Huelga, S. C., Kapeli, K., et al. (2016). Protein-RNA networks regulated by normal and ALS-associated mutant HNRNPA2B1 in the nervous system. Neuron 92, 780-795. doi: 10.1016/j.neuron.2016.09.050

Maruyama, H., Morino, H., Ito, H., Izumi, Y., Kato, H., Watanabe, Y., et al. (2010). Mutations of optineurin in amyotrophic lateral sclerosis. Nature 465, 223-226. doi: 10.1038 /nature 08971

Matus, S., Valenzuela, V., Medinas, D. B., and Hetz, C. (2013). ER dysfunction and protein folding stress in ALS. Int. J. Cell Biol. 2013:674751. doi: 10.1155/2013/674751

Moreno, J. A., Halliday, M., Molloy, C., Radford, H., Verity, N., Axten, J. M., et al. (2013). Oral treatment targeting the unfolded protein response prevents neurodegeneration and clinical disease in prion-infected mice. Sci. Transl. Med. 5:206ra138. doi: 10.1126/scitranslmed.3006767

Moreno, J. A., Radford, H., Peretti, D., Steinert, J. R., Verity, N., Martin, M. G., et al. (2012). Sustained translational repression by eIF $2 \alpha-P$ mediates prion neurodegeneration. Nature 485, 507-511. doi: 10.1038/nature11058

Ng, S.-Y., Soh, B. S., Rodriguez-Muela, N., Hendrickson, D. G., Price, F., Rinn, J. L., et al. (2015). Genome-wide RNA-seq of human motor neurons implicates selective ER stress activation in spinal muscular atrophy. Cell Stem Cell 17, 569-584. doi: 10.1016/j.stem.2015.08.003

Nonhoff, U., Ralser, M., Welzel, F., Piccini, I., Balzereit, D., Yaspo, M. L., et al. (2007). Ataxin-2 interacts with the DEAD/H-box RNA helicase DDX6 and interferes with P-bodies and stress granules. Mol. Biol. Cell 18, 1385-1396. doi: 10.1091/mbc.e06-12-1120

Protter, D. S., and Parker, R. (2016). Principles and properties of stress granules. Trends Cell Biol. 26, 668-679. doi: 10.1016/j.tcb.2016. 05.004

Radford, H., Moreno, J. A., Verity, N., Halliday, M., and Mallucci, G. R. (2015). PERK inhibition prevents tau-mediated neurodegeneration in a mouse model of frontotemporal dementia. Acta Neuropathol. 130, 633-642. doi: $10.1007 / \mathrm{s} 00401-015-1487-\mathrm{z}$

Ratti, A., and Buratti, E. (2016). Physiological functions and pathobiology of TDP-43 and FUS/TLS proteins. J. Neurochem. 138, 95-111. doi: 10.1111/jnc. 13625

Romano, M., Feiguin, F., and Buratti, E. (2016). TBPH/TDP-43 modulates translation of Drosophila futsch mRNA through an UG-rich sequence within its 5'UTR. Brain Res. 1647, 50-56. doi: 10.1016/j.brainres.2016.02.022

Rossi, S., Serrano, A., Gerbino, V., Giorgi, A., Di Francesco, L., Nencini, M., et al. (2015). Nuclear accumulation of mRNAs underlies G4C2-repeat-induced translational repression in a cellular model of C9orf72 ALS. J. Cell Sci. 128, 1787-1799. doi: $10.1242 /$ jcs. 165332

Ruegsegger, C., and Saxena, S. (2016). Proteostasis impairment in ALS. Brain Res. 1648, 571-579. doi: 10.1016/j.brainres.2016.03.032

Saxena, S., Cabuy, E., and Caroni, P. (2009). A role for motoneuron subtypeselective ER stress in disease manifestations of FALS mice. Nat. Neurosci. 12, 627-636. doi: 10.1038/nn.2297 
Shelkovnikova, T. A., Robinson, H. K., Southcombe, J. A., Ninkina, N., and Buchman, V. L. (2014). Multistep process of FUS aggregation in the cell cytoplasm involves RNA-dependent and RNA-independent mechanisms. Hum. Mol. Genet. 23, 5211-5226. doi: 10.1093/hmg/ddu243

Spriggs, K. A., Bushell, M., and Willis, A. E. (2010). Translational regulation of gene expression during conditions of cell stress. Mol. Cell 40, 228-237. doi: 10.1016/j.molcel.2010.09.028

Sun, S., Sun, Y., Ling, S. C., Ferraiuolo, L., McAlonis-Downes, M., Zou, Y., et al. (2015). Translational profiling identifies a cascade of damage initiated in motor neurons and spreading to glia in mutant SOD1-mediated ALS. Proc. Natl. Acad. Sci. U S A 112, E6993-E7002. doi: 10.1073/pnas.1520639112

Swinnen, B., and Robberecht, W. (2014). The phenotypic variability of amyotrophic lateral sclerosis. Nat. Rev. Neurol. 10, 661-670. doi: 10.1038/nrneurol.2014.184

Tao, Z., Wang, H., Xia, Q., Li, K., Jiang, X., Xu, G., et al. (2015). Nucleolar stress and impaired stress granule formation contribute to C9orf72 RAN translation-induced cytotoxicity. Hum. Mol. Genet. 24, 2426-2441. doi: 10.1093/hmg/ddv005

Taylor, J. P., Brown, R. H. Jr., and Cleveland, D. W. (2016). Decoding ALS: from genes to mechanism. Nature 539, 197-206. doi: 10.1038/nature20413

Wang, L., Popko, B., and Roos, R. P. (2011). The unfolded protein response in familial amyotrophic lateral sclerosis. Hum. Mol. Genet. 20, 1008-1015. doi: $10.1093 / \mathrm{hmg} / \mathrm{ddq} 546$

Wang, L., Popko, B., and Roos, R. P. (2014a). An enhanced integrated stress response ameliorates mutant SOD1-induced ALS. Hum. Mol. Genet. 23, 2629-2638. doi: 10.1093/hmg/ddt658

Wang, L., Popko, B., Tixier, E., and Roos, R. P. (2014b). Guanabenz, which enhances the unfolded protein response, ameliorates mutant SOD1-induced amyotrophic lateral sclerosis. Neurobiol. Dis. 71, 317-324. doi: 10.1016/j.nbd. 2014.08.010
Wen, X., Tan, W., Westergard, T., Krishnamurthy, K., Markandaiah, S. S., Shi, Y., et al. (2014). Antisense proline-arginine RAN dipeptides linked to C9ORF72-ALS/FTD form toxic nuclear aggregates that initiate in vitro and in vivo neuronal death. Neuron 84, 1213-1225. doi: 10.1016/j.neuron.2014. 12.010

Wen, X., Westergard, T., Pasinelli, P., and Trotti, D. (2017). Pathogenic determinants and mechanisms of ALS/FTD linked to hexanucleotide repeat expansions in the C9orf72 gene. Neurosci. Lett. 636, 16-26. doi: 10.1016/j. neulet.2016.09.007

Xu, Z., Poidevin, M., Li, X., Li, Y., Shu, L., Nelson, D. L., et al. (2013). Expanded GGGGCC repeat RNA associated with amyotrophic lateral sclerosis and frontotemporal dementia causes neurodegeneration. Proc. Natl. Acad. Sci. U S A 110, 7778-7783. doi: 10.1073/pnas.1219 643110

Yasuda, K., Zhang, H., Loiselle, D., Haystead, T., Macara, I. G., and Mili, S. (2013). The RNA-binding protein Fus directs translation of localized mRNAs in APC-RNP granules. J. Cell Biol. 203, 737-746. doi: 10.1083/jcb.2013 06058

Conflict of Interest Statement: The authors declare that the research was conducted in the absence of any commercial or financial relationships that could be construed as a potential conflict of interest.

Copyright (C) 2017 Cestra, Rossi, Di Salvio and Cozzolino. This is an open-access article distributed under the terms of the Creative Commons Attribution License (CC BY). The use, distribution and reproduction in other forums is permitted, provided the original author(s) or licensor are credited and that the original publication in this journal is cited, in accordance with accepted academic practice. No use, distribution or reproduction is permitted which does not comply with these terms. 